\title{
Dynamics modeling and performance analysis of underwater vehicles based on the Boltzmann-Hamel equations approach
}

\author{
Elżbieta Jarzębowska ${ }^{1, *}$, Michat Cichowski ${ }^{1}$ \\ ${ }^{1}$ Warsaw University of Technology, Power and Aeronautical Engineering Dept., 00-665 Warsaw, Nowowiejska 24st, Poland
}

\begin{abstract}
The paper addresses dynamics modeling of underwater vehicles, which are typical examples of variable mass and configuration mechanical systems. Specifically, we address inertia-based propelled vehicles, whose mass changes due to change of water amount in their water tanks and their configuration changes due to movable mass enabling maneuvers. They can be additionally subjected to constraints, which are imposed due to their desired performance, tracking pre-specified motions or are control constraints implying underactuation. The paper presents a new approach to variable mass and configuration systems modeling, which is based upon Boltzmann-Hamel equations derived in quasi-coordinates. The dynamics framework based upon quasi-coordinates results in dynamic models suitable for performance analysis and model-based control applications. It serves free or constrained variable mass and configuration systems modeling. The example researched in the paper is an underwater vehicle, which is purely inertia-based propelled. The theoretical development is illustrated with simulation studies of dynamical behavior and performance of the underwater vehicle model moving a specified trajectory.
\end{abstract}

\section{Introduction}

The paper addresses dynamics modeling of underwater vehicles (UV), which are variable mass and configuration mechanical systems. They can be additionally subjected to constraints, which are imposed due to their desired performance, tracking pre-specified motions or are control constraints implying underactuation. An UV is a typical example of variable mass and configuration system.

There are many systems whose mass or configuration change when they operate or a change of mass is their way of propulsion. The examples of such systems of engineering significance are ground mobile robots and manipulators, whose mass or configuration change due to carrying loads, excavating machines or others transporting various materials. Also, space vehicles are of interest in this context, since their service missions like refueling or debris removal are strongly related to control of their variable mass or configuration dynamics. If sloshing is to be taken into account for spacecraft, then a variable configuration dynamics is also welcome. All these variable mass systems mentioned above operate and deliver services so they are controlled systems for which reliable and computationally efficient dynamic models are needed.

Almost all modeling approaches to obtain either dynamics or control models are methods based upon Newton-Euler or Lagrange equations; see for example [1-3] and references there. Variable mass systems are of interest in mechanics for a long time and the beginning of its theory is attributed to Mieszczerskii [4] and the beginning of analytical mechanics of these systems is dated when Lagrange equations were derived for them [5]. Nowadays, research on variable mass systems is intensive and latest results with references to other works can be found in [6]. There, as in other works, holonomic systems with mass being mostly a time function are considered. Also, in e.g. like [1-3] and many other works, rigid body mechanics approach to variable mass and configuration systems is applied. Some preliminary results in modeling variable mass systems are presented in [7]. The results are promising so the main motivation of the paper is to develop a modeling framework for variable mass and configuration systems.

The paper presents a new approach to variable mass and configuration systems modeling, which is based upon Boltzmann-Hamel equations derived in quasicoordinates. The dynamics framework based upon quasicoordinates results in dynamics models suitable for performance analysis and model-based control applications. The paper contribution is then a development of dynamics modeling method based upon Boltzmann-Hamel equations derived in quasicoordinates. It serves free or constrained variable mass and configuration systems modeling. The example researched herein is an UV, which can be powered by internal batteries, is a purely inertia-based propelled or hybrid propelled, when additional batteries are added to facilitate or speed up its maneuvers. The theoretical development is illustrated with simulation studies of dynamical behavior and performance of an UV moving a specified trajectory.

The paper is organized as follows. Section 1 presents briefly an introduction about variable mass systems modeling methods. The theoretical framework, i.e. the variable mass and configuration system BoltzmannHamel motion equations are derived in Section 2. Section 3 presents simulation studies of the new equations applications to modeling an UV. The paper closes with conclusions and a list of references.

Corresponding author: elajarz@meil.pw.edu.pl 


\section{The Boltzmann-Hamel equations for variable mass and configuration systems}

\subsection{Underwater vehicle and external forces modeling}

The Boltzmann-Hamel based modeling framework is derived in this Section for a variable mass and configuration system. The system is represented herein by a UV model, which is inertia based propelled. It is quite a general example of a system of variable mass water amount in its two water tanks changes. Also, it is equipped with a movable mass that may move back and forth and asides enabling the vehicle maneuvers.

The UV has 9 degrees of freedom, i.e. 3 translations and 3 rotations for the vehicle, and 3 translations for movable ballast mass. It is equipped with two water tanks, the front one and the rear one, whose water amount can be controlled due to the vehicle motion mode. The scheme of a vehicle is presented in Fig. 1.

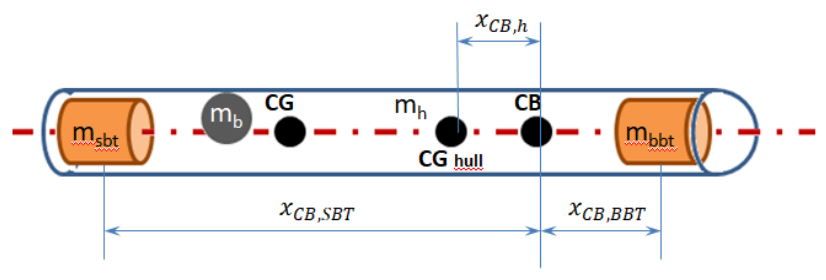

Fig. 1. Inertia and geometry parameters of the UV model.

The original Boltzmann-Hamel motion equations for constant mass systems are derived in quasi-coordinates and quasi-velocities [7]. They are non-inertial coordinates and their selection is presented in Fig. 2. They are velocities $U, V, W$ along the body fixed frame. Rotations about these axes are described by angular velocities $P, Q, R$.

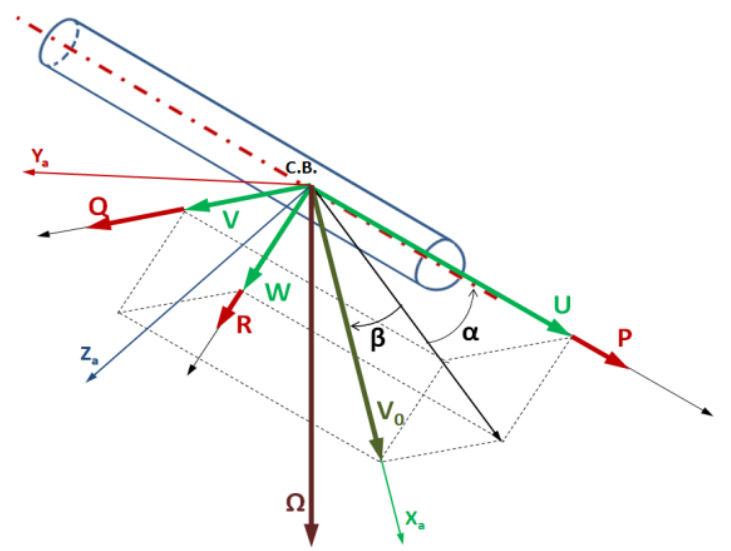

Fig. 2. The body fixed frame and quasi-velocities describing motion of the UV.

The UV model geometry and mass data is gathered in Table 1.
Table 1. UV geometry and mass data.

$$
\begin{array}{ll}
x_{C B, S B T}=0.5 \mathrm{~m} & \begin{array}{l}
\text { distance from CB } \\
\text { to stern ballast tank }
\end{array} \\
x_{C B, B B T}=0.5 \mathrm{~m} & \begin{array}{l}
\text { distance from CB } \\
\text { tom bow ballast tank }
\end{array} \\
x_{C B, h}=0 & \begin{array}{l}
\text { distance from CB } \\
\text { to center of gravity of hull }
\end{array} \\
m_{h}=34.6 \mathrm{~kg} & \text { mass of hull } \\
m_{p}=0.4 \mathrm{~kg} & \text { mass of movable ballast } \\
m_{\text {disp }}=35 \mathrm{~kg} & \text { displacement } \\
\text { Moments of inertia of hull (principal) } \\
I_{x x}=0.172 \mathrm{~kg} \cdot \mathrm{m}^{2} \\
I_{y y}=5.919 \mathrm{~kg} \cdot \mathrm{m}^{2} \\
I_{z z}=5.919 \mathrm{~kg} \cdot \mathrm{m}^{2}
\end{array}
$$

The buoyancy and gravity forces that act upon the vehicle are presented by equation (1). It is assumed that the UV can move with moderate velocities so hydrodynamic forces are determined according to classical fluid dynamics as in (2) and Table 2.

$$
\begin{aligned}
& \bar{F}_{G, C B}+\bar{F}_{B, C B}=\left[\begin{array}{ccc}
\mathbb{1}_{3 x 3} & 0_{3 x 3} & 0_{3 x 3} \\
0_{3 x 3} & \tilde{r}_{C B, C M} & 0_{3 x 3} \\
0_{3 x 3} & 0_{3 x 3} & 0_{3 x 3}
\end{array}\right]\left(\left[\begin{array}{c}
\Lambda_{\nu} \\
\Lambda_{\nu} \\
0_{3 x 3}
\end{array}\right]\left[\begin{array}{c}
0 \\
0 \\
M g
\end{array}\right]+\left[\begin{array}{c}
\Lambda_{\nu} \\
0_{3 x 3} \\
0_{3 x 3}
\end{array}\right]\left[\begin{array}{c}
0 \\
0 \\
-\rho v g
\end{array}\right]\right)= \\
& =\left[\begin{array}{ccc}
\mathbb{1}_{3 x 3} & 0_{3 x 3} & 0_{3 x 3} \\
0_{3 x 3} & \tilde{r}_{C B, C M} & 0_{3 x 3} \\
0_{3 x 3} & 0_{3 x 3} & 0_{3 x 3}
\end{array}\right]\left[\begin{array}{c}
-\sin \theta(M g-\rho v g) \\
\cos \theta \sin \phi(M g-\rho v g) \\
\sin \theta \sin \phi(M g-\rho v g) \\
-\sin \theta M g \\
\cos \theta \sin \phi M g \\
\sin \theta \sin \phi M g \\
0 \\
0 \\
0
\end{array}\right]=\left[\begin{array}{c}
\sin \theta(M g-\rho v g) \\
\cos \theta \sin \phi(M g-\rho v g) \\
\cos \theta \cos \phi(M g-\rho v g) \\
\cos \theta\left(y_{C B, C M} \cos \phi-z_{C B, C M} \sin \phi\right) M g \\
-\left(z_{C B, C M} \sin \theta+x_{C B, C M} \cos \theta \cos \phi\right) M g \\
\left(y_{C B, C M} \sin \theta+x_{C B, C M} \cos \theta \sin \phi\right) M g \\
0 \\
0 \\
0
\end{array}\right] \\
& \bar{F}_{H, C B}^{V}=\left[\begin{array}{c}
X_{H, C B}^{V} \\
Y_{H, C B}^{V} \\
Z_{H, C B}^{V} \\
L_{H, C B}^{V} \\
M_{H, C B}^{V} \\
N_{H, C B}^{V} \\
0 \\
0 \\
0
\end{array}\right]=\left[\begin{array}{c}
-K_{D 0}-K_{D}\left(\alpha^{2}+\beta^{2}\right) \\
K_{\beta} \beta \\
-K_{\alpha} \alpha \\
-K_{P} P \\
--K_{M} \alpha-K_{Q} Q \\
K_{M Y} \beta-K_{R} R \\
0 \\
0 \\
0
\end{array}\right] V_{0}^{2}
\end{aligned}
$$

Table 2. Hydrodynamic force coefficients for the UV model in the velocity related frame.

$$
\begin{aligned}
& K_{d 0}=7.19 \frac{\mathrm{kg}}{\mathrm{m}} \\
& K_{d}=386.29 \frac{\mathrm{kg}}{\mathrm{m} \cdot \mathrm{rad}} \mathrm{d}^{2} \\
& K_{\beta}=115.65 \frac{\mathrm{kg}}{\mathrm{m} \cdot \mathrm{rad}} \\
& K_{\alpha}=440.99 \frac{\mathrm{kg}}{\mathrm{m} \cdot \mathrm{rad}} \\
& K_{p}=190.83 \frac{\mathrm{kg} \cdot \mathrm{r}}{\mathrm{rad}} \\
& K_{m}=65.84 \frac{\mathrm{kg}}{\mathrm{rad}} \\
& K_{q}=205.64 \frac{\mathrm{kg} \cdot \mathrm{s}}{\mathrm{rad}} \\
& K_{m y}=34.1 \frac{\mathrm{kg}}{\mathrm{ra} d} \\
& K_{r}=389.3 \frac{\mathrm{kg} \cdot \mathrm{s}}{\mathrm{rad}^{2}}
\end{aligned}
$$




\subsection{Boltzmann-Hamel equations in the matrix form}

The original Boltzmann-Hamel equations for constant mass systems have the form, see e.g. [7] and references there

$$
\frac{d}{d t}\left(\frac{\partial T}{\partial \omega_{j}}\right)-\frac{\partial T}{\partial \pi_{j}}+\sum_{l=1}^{n} \sum_{\alpha=1}^{n} \gamma_{j \alpha}^{l} \frac{\partial T}{\partial \omega_{l}} \omega_{\alpha}=F_{j}
$$

with the Boltzmann-Hamel symbols of the form

$$
\gamma_{j \alpha}^{l}=\sum_{\sigma=1}^{n} \sum_{\lambda=1}^{n}\left(\frac{\partial a_{l, \sigma}}{\partial q_{\lambda}}-\frac{\partial a_{l, \lambda}}{\partial q_{\sigma}}\right) b_{\sigma, j} b_{\lambda, \alpha}
$$

Advantages of the Boltzmann-Hamel equations are multiple. They are first order differential equations in quasi-velocities, which do not have to possess physical interpretations and can be selected by a designer or a control engineer. Also, the kinematic constraint equations can be easily eliminated via the quasivelocities selection and the forces acting and interacting in the system can be easily determined [7].

Let us write the Boltzmann-Hamel equations (3) in a matrix form applying the following notation

$$
\frac{d}{d t}\left[\begin{array}{c}
\frac{\partial T}{\partial \omega_{1}} \\
\frac{\partial T}{\partial \omega_{2}} \\
\vdots \\
\frac{\partial T}{\partial \omega_{n}}
\end{array}\right]-\left[\begin{array}{c}
\frac{\partial T}{\partial \pi_{1}} \\
\frac{\partial T}{\partial \pi_{2}} \\
\vdots \\
\frac{\partial T}{\partial \pi_{n}}
\end{array}\right]+\sum_{\alpha=1}^{n} \omega_{\alpha}\left[\begin{array}{c}
\sum_{l=1}^{n} \gamma_{1}^{l} \alpha \frac{\partial T}{\partial \omega_{l}} \\
\sum_{l=1}^{n} \gamma_{2}^{l} \alpha \frac{\partial T}{\partial \omega_{l}} \\
\vdots \\
\sum_{l=1}^{n} \gamma_{n}^{l} \alpha \frac{\partial T}{\partial \omega_{l}}
\end{array}\right]=\left[\begin{array}{c}
F_{1} \\
F_{2} \\
\vdots \\
F_{n}
\end{array}\right]
$$

$$
\sum_{\alpha=1}^{n} \omega_{\alpha}\left[\begin{array}{c}
\sum_{l=1}^{n} \gamma_{1}^{l} \alpha \frac{\partial T}{\partial \omega_{l}} \\
\sum_{l=1}^{n} \gamma_{2}^{l} \alpha \frac{\partial T}{\partial \omega_{l}} \\
\vdots \\
\sum_{l=1}^{n} \gamma_{n}^{l} \frac{\partial T}{\partial \omega_{l}}
\end{array}\right]=\sum_{\alpha=1}^{n}\left(\omega_{\alpha}\left[\begin{array}{cccc}
\gamma_{1 \alpha}^{1} & \gamma_{1 \alpha}^{2} & \cdots & \gamma_{1 \alpha}^{n} \\
\gamma_{2 \alpha}^{1} & \gamma_{2 \alpha}^{2} & \cdots & \gamma_{2 \alpha}^{n} \\
\vdots & \vdots & \ddots & \vdots \\
\gamma_{n \alpha}^{1} & \gamma_{n \alpha}^{2} & \cdots & \gamma_{n \alpha}^{n}
\end{array}\right]\left[\begin{array}{c}
\frac{\partial T}{\partial \omega_{1}} \\
\frac{\partial T}{\partial \omega_{2}} \\
\vdots \\
\frac{\partial T}{\partial \omega_{n}}
\end{array}\right]\right)
$$

Also, introducing the notation

$$
\Gamma_{\alpha}=\left[\begin{array}{cccc}
\gamma_{1 \alpha}^{1} & \gamma_{1 \alpha}^{2} & \cdots & \gamma_{1 \alpha}^{n} \\
\gamma_{2 \alpha}^{1} & \gamma_{2 \alpha}^{2} & \cdots & \gamma_{2 \alpha}^{n} \\
\vdots & \vdots & \ddots & \vdots \\
\gamma_{n \alpha}^{1} & \gamma_{n \alpha}^{2} & \cdots & \gamma_{n \alpha}^{n}
\end{array}\right]
$$

and

$$
G(\omega)=\sum_{\alpha=1}^{n} \Gamma_{\alpha} \omega_{\alpha}
$$

the matrix form of equations (3) can be presented as

$$
\frac{d}{d t}\left(\frac{\partial T}{\partial \bar{\omega}}\right)-\frac{\partial T}{\partial \bar{\pi}}+G(\bar{\omega}) \frac{\partial T}{\partial \bar{\omega}}=\bar{F}_{e x t}
$$

The matrix form (6) of the Boltzmann-Hamel equations is needed for derivation of the general modeling framework based upon these equations, for which mass and configuration can change or be constant.

\subsection{Boltzmann-Hamel equations for variable mass and configuration systems}

The derivation of the Boltzmann-Hamel equations for variable mass and configuration systems starts from the presentation of the system kinetic energy using matrix and vector notation. It enables easy handling of mass and inertia terms in the system model equations. The general form of the kinetic energy for the variable mass and configuration system is given by (7), where the mass matrix is composed of

$$
\begin{gathered}
M=m_{h}+m_{p}+m_{b b c}+m_{s b s} \\
M \bar{r}_{C B, C M}=m_{h} \bar{r}_{C B, h}+m_{p} \bar{r}_{C B, p}+m_{b b c} \bar{r}_{C B, b b c}+m_{s b c} \bar{r}_{C B, s b c} \\
\mathbb{I}=\mathbb{I}_{h, C M}+m_{h} \widetilde{r}_{C B, h} \widetilde{r}_{C B, h}^{T}+m_{p} \widetilde{r}_{C B, p} \widetilde{r}_{C B, p}^{T}+ \\
+m_{b b c} \widetilde{r}_{C B, b b c} \widetilde{r}_{C B, b b c}^{T}+m_{s b c} \widetilde{r}_{C B, s b c} \widetilde{r}_{C B, s b c}^{T}
\end{gathered}
$$

Vectors $\bar{\omega}_{v}$ and $\bar{\omega}_{\Omega}$ stand for the linear velocity vector and the angular velocity vector, both in the body fixed frame. The other vectors are:

$-\bar{r}_{C B, p}=\left[\begin{array}{lll}r_{p x} & r_{p y} & r_{p z}\end{array}\right]^{T}$ is a distance of a movable mass from the buoyancy center,

$-\bar{r}_{C B, b b c}=\left[\begin{array}{lll}x_{C B, b b c} & 0 & 0\end{array}\right]^{T}$ is a position vector of the front water tank,

$-\bar{r}_{C B, s b c}=\left[\begin{array}{lll}x_{C B, s b c} & 0 & 0\end{array}\right]^{T}$ is a position vector of the rear water tank,

- vectors with the top wave symbol denote the skewsymmetry matrices, e.g.:

$$
\tilde{\omega}_{\Omega}=\left[\begin{array}{ccc}
0 & -\omega_{\Omega z} & \omega_{\Omega y} \\
\omega_{\Omega z} & 0 & -\omega_{\Omega x} \\
-\omega_{\Omega y} & \omega_{\Omega x} & 0
\end{array}\right]
$$

The matrix of the Boltzmann-Hamel coefficients can be written as

$$
G=\left[\begin{array}{ccccccccc}
0 & -R & Q & 0 & 0 & 0 & 0 & 0 & 0 \\
R & 0 & -P & 0 & 0 & 0 & 0 & 0 & 0 \\
-Q & P & 0 & 0 & 0 & 0 & 0 & 0 & 0 \\
0 & -W & V & 0 & -R & Q & 0 & 0 & 0 \\
W & 0 & -U & R & 0 & -P & 0 & 0 & 0 \\
-V & U & 0 & -Q & P & 0 & 0 & 0 & 0 \\
0 & 0 & 0 & 0 & 0 & 0 & 0 & 0 & 0 \\
0 & 0 & 0 & 0 & 0 & 0 & 0 & 0 & 0 \\
0 & 0 & 0 & 0 & 0 & 0 & 0 & 0 & 0
\end{array}\right]=\left[\begin{array}{cccc}
\widetilde{\omega}_{\Omega} & 0_{3 x 3} & 0_{3 x 3} \\
\widetilde{\omega}_{\nu} & \widetilde{\omega}_{\Omega} & 0_{3 x 3} \\
0_{3 x 3} & 0_{3 x 3} & 0_{3 x 3}
\end{array}\right]
$$

Finally, determining 


$$
\frac{d}{d t}\left(\frac{\partial T}{\partial \bar{\omega}}\right)=\frac{d}{d t} \frac{\partial}{\partial \bar{\omega}}\left(\frac{1}{2} \bar{\omega}^{T} \mathbb{M} \bar{\omega}\right)=\frac{d}{d t}(\mathbb{M} \bar{\omega})=\mathbb{M} \dot{\bar{\omega}}+\dot{\mathbb{M}} \bar{\omega}
$$

with the inertia matrix derivative given by (12), the Boltzmann-Hamel equations have the form (13). They can be rewritten in the form (14), which clearly shows the inertia components in the system. Equations (14) are

$$
\begin{aligned}
& T=\frac{1}{2}\left[\begin{array}{lll}
\bar{\omega}_{\nu}^{T} & \bar{\omega}_{\Omega}^{T} & \dot{\bar{r}}_{C B, p}^{T}
\end{array}\right]\left[\begin{array}{ccc}
M \mathbb{1}_{3 x 3} & M \tilde{r}_{C B, C M}^{T} & m_{p} \mathbb{1}_{3 x 3} \\
M \tilde{r}_{C B, C M} & \mathbb{I} & m_{p} \widetilde{r}_{C B, p} \\
m_{p} \mathbb{1}_{3 x 3} & m_{p} \tilde{r}_{C B, p}^{T} & m_{p} \mathbb{1}_{3 x 3}
\end{array}\right]\left[\begin{array}{c}
\bar{\omega}_{\nu} \\
\bar{\omega}_{\Omega} \\
\dot{\bar{r}}_{C B, p}
\end{array}\right]=\frac{1}{2} \bar{\omega}^{T} \mathbb{M} \bar{\omega} \\
& \dot{\mathbb{M}}=\left[\begin{array}{ccc}
\left(\dot{m}_{b b c}+\dot{m}_{s b c}\right) \mathbb{1}_{3 x 3} & \dot{m}_{b b c} \tilde{r}_{C B, b b c}^{T}+\dot{m}_{s b c} \widehat{r}_{C B, s b c}^{T} & 0_{3 x 3} \\
\dot{m}_{b b c} \widetilde{r}_{C B, b b c}+\dot{m}_{s b c} \widetilde{r}_{C B, s b c} & \dot{m}_{b b c} \widetilde{r}_{C B, b b c} \widetilde{r}_{C B, b b c}^{T}+\dot{m}_{s b c} \widetilde{r}_{C B, s b c} \widetilde{r}_{C B, s b c}^{T} & 0_{3 x 3} \\
0_{3 x 3} & 0_{3 x 3} & 0_{3 x 3}
\end{array}\right]+ \\
& +\left[\begin{array}{ccc}
0_{3 x 3} & m_{p} \dot{\vec{r}}_{C B, p}^{T} & 0_{3 x 3} \\
m_{p} \dot{\tilde{r}}_{C B, p} & m_{p}\left(\dot{\widetilde{r}}_{C B, p} \tilde{r}_{C B, p}^{T}+\tilde{r}_{C B, p} \dot{\vec{r}}_{C B, p}\right) & m_{p} \dot{\widetilde{r}}_{C B, p} \\
0_{3 x 3} & m_{p} \dot{\vec{r}}_{C B, p}^{T} & 0_{3 x 3}
\end{array}\right] \\
& \mathbb{M} \dot{\bar{\omega}}+\left(\left[\begin{array}{ccc}
\widetilde{\omega}_{\Omega} & 0_{3 x 3} & 0_{3 x 3} \\
\widetilde{\omega}_{\nu} & \widetilde{\omega}_{\Omega} & \tilde{\tilde{r}}_{C B, p} \\
0_{3 x 3} & 0_{3 x 3} & \widetilde{\omega}_{\Omega}
\end{array}\right] \mathbb{M}+\mathbb{M}\left[\begin{array}{ccc}
0_{3 x 3} & 0_{3 x 3} & 0_{3 x 3} \\
0_{3 x 3} & 0_{3 x 3} & 0_{3 x 3} \\
0_{3 x 3} & \dot{\tilde{r}}_{C B, p}^{T} & 0_{3 x 3}
\end{array}\right]+\dot{\mathbb{M}}_{b b c}+\dot{\mathbb{M}}_{s b c}\right) \bar{\omega}=\bar{F} \\
& \mathbb{M} \dot{\bar{\omega}}+\left[\begin{array}{ccc}
\tilde{\omega}_{\Omega} M & \tilde{\omega}_{\Omega} M \tilde{r}_{C B, C M}^{T} & 2 \tilde{\omega}_{\Omega} m_{p} \\
M \tilde{r}_{C B, C M} \tilde{\omega}_{\Omega} & \tilde{\omega}_{\Omega} \mathbb{I} & 2 m_{p} \tilde{r}_{C B, p} \widetilde{\omega}_{\Omega} \\
\tilde{\omega}_{\Omega} m_{p} & \tilde{\omega}_{\Omega} m_{p} \tilde{r}_{C B, p}^{T} & 2 \tilde{\omega}_{\Omega} m_{p}
\end{array}\right] \bar{\omega}+\left(\dot{\mathbb{M}}_{b b c}+\dot{\mathbb{M}}_{s b c}\right) \bar{\omega}=\bar{F}_{e x t}
\end{aligned}
$$

\section{Simulation studies - inertia propelled UV dynamics and performance}

In this Section, using the general modeling framework for variable mass and configuration systems based upon the Boltzmann-Hamel equations, dynamics and performance of inertia propelled UV is presented. Two UV motion scenarios are selected. One presents the typical saw-tooth motion of the UV and the second, a spiral motion. Both motions are controlled in the openloop through the control of the movable mass motion and the regulation of water amount in the water tanks.

\subsection{Scenario 1- saw-tooth motion of the UV}

The saw-tooth UV motion is its typical motion pattern. The movable mass does not move but water in the water tanks is regulated in a sinusoidal pattern with a period $\mathrm{T}=30 \mathrm{~s}$ as shown in Fig. 3. The UV initial motion conditions are $\mathrm{U}=0.05 \mathrm{~m} / \mathrm{s}$.
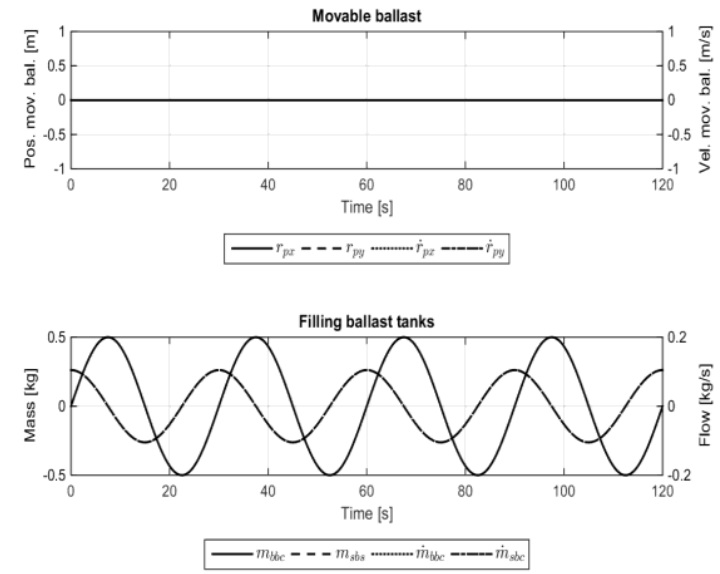

Fig. 3. The movable mass and changes of water amount in the tanks in the saw-tooth UV motion. 


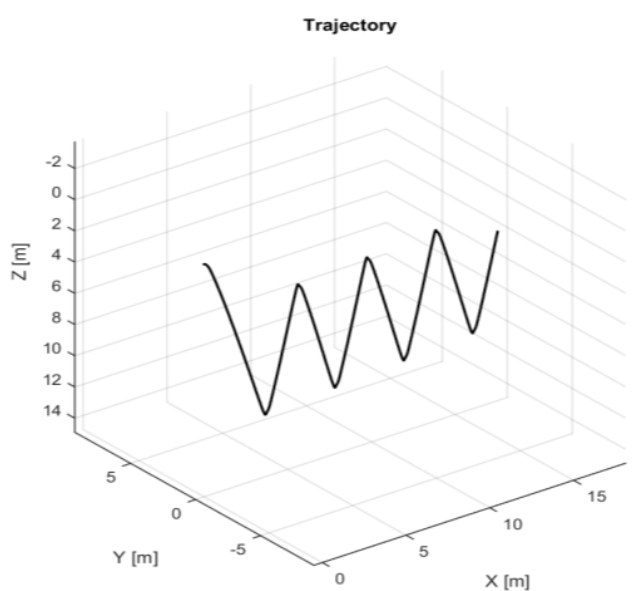

Fig. 4. The UV saw-tooth motion.

The UV velocities $U, V, W$, the total velocity $V_{0}$ and the angular velocities $\mathrm{P}, \mathrm{Q}, \mathrm{R}$ are depicted in Fig. 5.
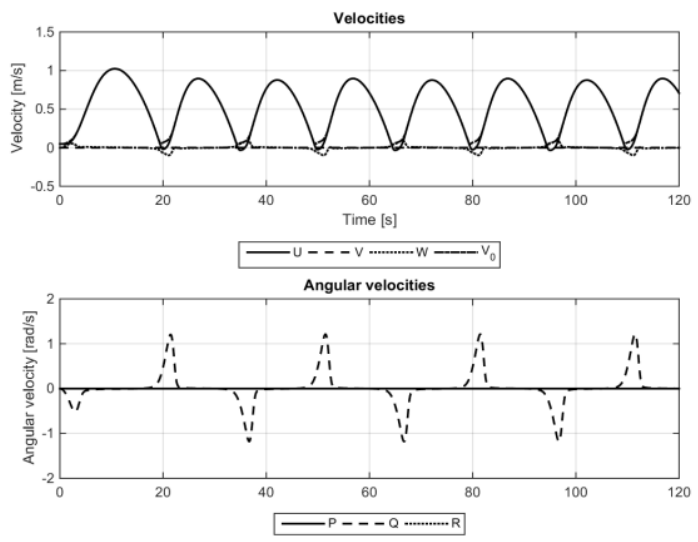

Fig. 5. The body fixed frame and quasi-velocities describing motion of the UV.

\subsection{Scenario 2- spiral motion of the UV}

In this scenario the UV is to move a spiral motion down into the water. The UV configuration is as follows: motion starts from rest, the water tanks are filled with water of $0.1 \mathrm{~kg}$ each. The movable mass is located at $0.05 \mathrm{~m}$ to stern and $0.1 \mathrm{~m}$ to starboard. Initial motion conditions are selected to be: $\mathrm{U}=0.05 \mathrm{~m} / \mathrm{s}$, simulation time is $\mathrm{t}=1200 \mathrm{sec}$.

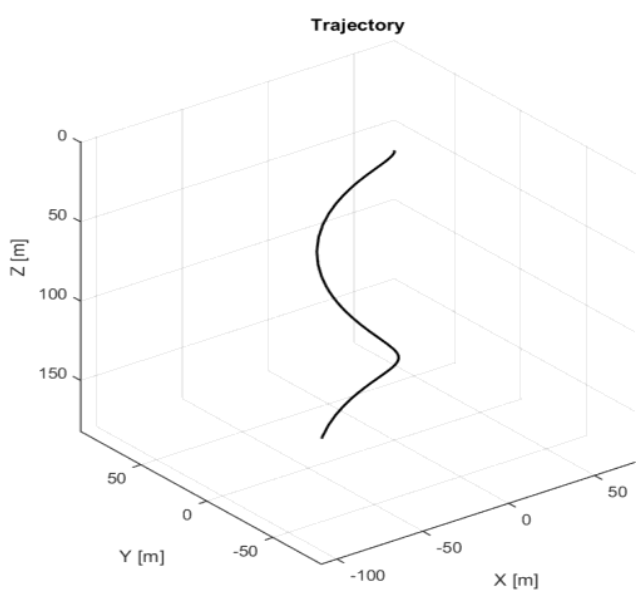

Fig. 6. The body fixed frame and quasi-velocities describing motion of the UV.
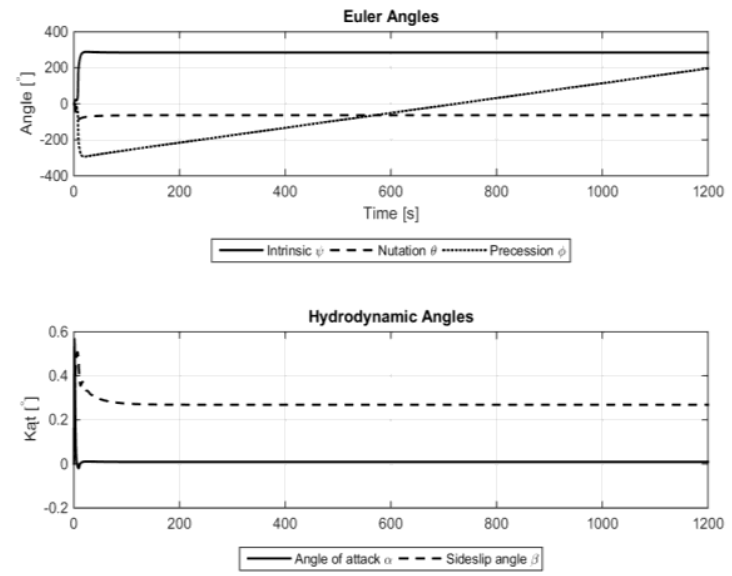

Fig. 7. The body fixed frame and quasi-velocities describing motion of the UV.

Other motions along other trajectories can be simulated for the UV in the same way.

\section{Conclusions}

The paper presents a new method of variable mass and configuration systems modeling, which is based upon the Boltzmann-Hamel equations derived in quasicoordinates.

The presented dynamics framework based upon quasi-coordinates results in dynamic models suitable for performance analysis and model-based control applications. The dynamics framework serves free or constrained constant and/or variable mass and configuration systems modeling. The example of an underwater, inertia-based propelled vehicle model illustrates the theoretical development of variable mass and configuration systems dynamics. Simulation studies of the dynamical behavior and performance of the underwater vehicle moving a specified trajectory illustrate the framework applications.

Future research plans to incorporate kinematic constraints into the framework and apply it to design model-based control strategies for the UV. 


\section{References}

1. A. Bender, Analysis of an Autonomous Underwater Glider, Australian Centre for Field Robotics, Univ. Sydney, Sydney, NSW (2006).

2. J. G. Graver, Underwater Gliders: Dynamics, Control and Design, Ph.D. Thesis, Princeton University (2005).

3. S. Guo, X. Lin, Development of a vectored water-jetbased spherical underwater vehicle, in: Nuno Cruz (Ed.), Autonomous Underwater Vehicles, Intech (2011).

4. I. V. Mieszczerskii, Motion Equations of a Variable Mass Particle in a General Case - Works on Mechanics of Variable Mass Bodies. Gostiechisdat, (in Russian), Moscow (1949).

5. A. A. Kosmodemianskii, Lectures in Mechanics of Bodies with Variable Mass, Uczionnye zapiski M.G.U. 154, Mechanics (1951).

6. L. Cveticanin, Dynamics of Bodies with TimeVariable Mass. Springer, Heidelberg (2016).

7. E. Jarzębowska, Model Based Tracking Control of Nonlinear Systems, Taylor and Francis Group, Boca Raton, 2012. 